\title{
Dynamics and quantumness of excitation energy transfer through a complex quantum network
}

\author{
M. Qin, ${ }^{1}$ H. Z. Shen, ${ }^{1}$ X. L. Zhao, ${ }^{1}$ and X. X. Yi*2 \\ ${ }^{1}$ School of Physics and Optoelectronic Technology, \\ Dalian University of Technology, Dalian 116024, China \\ ${ }^{2}$ Center for Quantum Sciences and School of Physics, \\ Northeast Normal University, Changchun 130024, China
}

(Dated: August 29, 2018)

\begin{abstract}
Understanding the mechanisms of efficient and robust energy transfer in organic systems provides us with new insights for the optimal design of artificial systems. In this paper, we explore the dynamics of excitation energy transfer (EET) through a complex quantum network by a toy model consisting of three sites coupled to environments. We study how the coherent evolution and the noiseinduced decoherence work together to reach efficient EET and illustrate the role of the phase factor attached to the coupling constant in the EET. By comparing the differences between the Markovian and non-Markovian dynamics, we discuss the effect of environment and the spatial structure of system on the dynamics and the efficiency of EET. A intuitive picture is given to show how the exciton is transferred through the system. Employing the simple model, we show the robustness of EET efficiency under the influence of the environment and elucidate the important role of quantum coherence in EET. We go further to study the quantum feature of the EET dynamics by quantumness and show the importance of quantum coherence from a new respect. We calculate the energy current in the EET and its quantumness, results for different system parameters are presented and discussed.

PACS numbers: 05.60.Gg, 03.65.Yz, 03.67.-a, 71.35.-y
\end{abstract}

\section{INTRODUCTION}

The processes of energy and information transfer in quantum networks play an important role in many areas of physics, chemistry and biology. They have been identified as central to quantum mechanics since its early days [1]. Under realistic physical conditions, the coupling of quantum system to environment is usually unavoidable, which leads to the deterioration of performance for fundamental mechanical processes in systems such as computing devices and biological organisms. Recent experiments, however, find evidence of long-lived quantum coherence in conditions that are often defined as hot and wet [2, 3] in the Fenna-Matthews-Olson (FMO) protein complex of the green-sulfur bacterium Chlorobiumtepidum and in the reaction center of the purple bacterium Rhodobactersphaeroides [4 10 ].

Inspired by these experimental observations, quantum coherence across multiple chromophoric sites has been suggested to play a significant role in achieving the remarkable efficient EET in FMO and other pigmentprotein complexes [11 13]. Since then, many works analyzing energy transport in these systems have been carried out 14 30], such as by the quantum network model [14 23], by the hierarchic equation [24 27], by the renormalization group [28, 29], and by semiquantum theory [30]. Theoretical investigations found that dephasing does not always hinder the efficiency of EET compared with perfectly quantum coherent system, which is

\footnotetext{
${ }^{*}$ Corresponding address: yixx@nenu.edu.cn
}

contrary to the conventional intuition 31]. The interplay of the quantum coherent evolution and dephasing contributes to the highly effective EET in light harvesting complexes [14, 32]. Understanding the underlying mechanism of such a process may assist us in designing novel nanofabricated structures for quantum transport and optimized solar cells.

Although there are many progresses made in this field, the interplay of the quantum coherent evolution and the relaxation process, however, has not been studied in the Haken-Strobl model, in which the energy relaxations are not included. In this paper, we introduce the relaxation process into the decoherence terms, and study the dynamics and the transfer efficiency in a complex network. We obtain several analytical solutions to illuminate how the coherent evolution and the noise-induced decoherence processes, especially the relaxation process, work together to reach efficient EET.

Besides, the experimental evidence has shown that the space distribution of the pigments is very important for the EET [5, 6]. Thus it is essential to take phase factors in the site-to-site couplings into account. We show how the spatial structure of the system, embodied in the site-to-site couplings [15, 33], affect the efficiency of the EET. We also take into account the effect of the quantum nature of the environment, such as Markovianity and non-Markovianity on the dynamics [22, 35 37]. Comparing the result of numerical calculation with recent experimental results, we analyze the effect of surrounding environment and inner spatial structure on the EET dynamics and efficiency. We find that the EET efficiency is robust against various environmental parame- 
ters. To shed more light on the quantum coherence that has been studied in many literatures in terms of entanglement [12, 24, 38 43], we apply a recently developed measure for quantum coherence called quantumness [44 46] to study the quantumness of the EET within the single excitation subspace. By calculating the energy current of the transfer dynamics and its quantumness, we show that quantum coherence indeed play an important role in the energy transfer.

The remainder of the paper is organized as follows. In Sec. II, we introduce a model to describe the complex quantum network, in which a phase factor is added into the coupling constant. This phase factor may come from the spatial structure of the light harvesting system. In Sec. III, we introduce a Markovian master equation including the relaxation, dephasing and dissipation to describe the network. Then we apply the fully connected network to derive an exact expression for the EET efficiency. The dependence of the EET on the initial state and the phase factor in the model are discussed in details. In Sec. IV, we focus on the Markovian and nonMarkovian effects in the dynamics. The effects of the phase factor and temperature on the dynamics are also discussed. In Sec. V, we calculate the energy current in the system and discuss the quantum coherent nature of the dynamics. The effect of the phase factor and temperature on the current and quantumness are explored. Sec. VI is devoted to concluding remarks.

\section{MODEL}

We discuss a network of three sites, two of them $|1\rangle$ and $|2\rangle$ may support excitations, which can hop from one site to the other (see Fig. 1). The two sites interact with the environment, and one of the sites (say site 2) is connected to the third site, sink $|3\rangle$. Once excitations fall into the sink, they can not escape. This network is a model simplified from the structure of the FMO complex, a network of seven coupled sites, each of which can be treated as a two-level system. The total Hamiltonian in the single exciton manifold includes three parts, $H=$ $H_{S}+H_{S B}+H_{B}$. The system part is given by,

$$
H_{S}=\varepsilon_{1} \sigma_{1}^{+} \sigma_{1}^{-}+\varepsilon_{2} \sigma_{2}^{+} \sigma_{2}^{-}+J\left(e^{-i \varphi} \sigma_{1}^{+} \sigma_{2}^{-}+e^{i \varphi} \sigma_{2}^{+} \sigma_{1}^{-}\right)
$$

where $\sigma_{j}^{+}$and $\sigma_{j}^{-}$are the raising and lowering operators for site $j$, defined by $\sigma_{j}^{+}=|j\rangle\langle 0|$ and $\sigma_{j}^{-}=|0\rangle\langle j|$, where $|0\rangle$ denotes the zero exciton state of the system and $|j\rangle$ represents one excitation on site $j . \varepsilon_{j}$ is the on-site energy of site $j$, and $J_{i j}$ is designated as the excitonic coupling between site $i$ and $j$, where $J_{i j}=J_{j i}=J$ is assumed. A phase factor $e^{-i \varphi}$ with $\varphi$ a real number is added to the inter-site coupling $J$, making the coupling constant complex.

The Hamiltonian of the environment, which modulates site energy fluctuation and brings decoherence, is usually

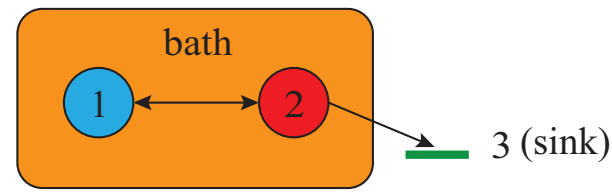

FIG. 1: (Color online) The schematic representation of three sites network. The exciton, initially in site $|1\rangle$, is transferred to site $|2\rangle$ and finally trapped by the sink $|3\rangle$.

modeled as a set of quantum oscillators,

$$
H_{B}=\sum_{j} \sum_{m=1,2} \omega_{m, j} b_{m, j}^{\dagger} b_{m, j}
$$

where $b_{m, j}^{\dagger}\left(b_{m, j}\right)$ is the creation (annihilation) operator of the $j$-th mode with frequency $\omega_{j}$ in the bath, we suppose that each site is separately coupled with its own environment. Thus the system-environment interection Hamiltonian reads [38]

$$
H_{S B}=\sum_{j} \sum_{m=1,2} g_{m, j}\left(b_{m, j}^{\dagger}+b_{m, j}\right)|m\rangle\langle m|,
$$

where $g_{m, j}$ denotes the coupling constant of the $m$-th site to its bath mode $j$.

The transport dynamics of a single excitation is described by a master equation that includes coherent evolution, relaxation, dissipation and dephasing. In addition, the exciton can be trapped in the sink. Once the excitation is trapped, it can not escape.

\section{EET DYNAMICS IN MARKOVIAN CASE}

Consider a Markovian environment, the dynamics of the reduced density matrix of the system is given by

$$
\begin{aligned}
\dot{\rho}(t)= & -i\left[H_{S}, \rho\right]+L_{\kappa}(\rho(t)) \\
& +L_{\gamma}(\rho(t))+L_{\Gamma}(\rho(t))+L_{s}(\rho(t)),
\end{aligned}
$$

where the Liouvilian on the right-hand side in Eq. (4) describes coherent dynamics, relaxation, dephasing, dissipation, and sink, respectively.

The relaxation (with rate $\Gamma_{12}\left(\Gamma_{21}\right)$ ) is described by Lindblad super-operators $L_{\kappa}$ [22, 37],

$$
\begin{aligned}
L_{\kappa}(\rho(t))= & \Gamma_{12}\left[\sigma_{2}^{+} \sigma_{1}^{-} \rho(t) \sigma_{1}^{+} \sigma_{2}^{-}\right. \\
& \left.-\frac{1}{2}\left\{\rho(t), \sigma_{1}^{+} \sigma_{2}^{-} \sigma_{2}^{+} \sigma_{1}^{-}\right\}\right] \\
& +\Gamma_{21}\left[\sigma_{1}^{+} \sigma_{2}^{-} \rho(t) \sigma_{2}^{+} \sigma_{1}^{-}\right. \\
& \left.-\frac{1}{2}\left\{\rho(t), \sigma_{2}^{+} \sigma_{1}^{+} \sigma_{1}^{-} \sigma_{2}^{-}\right\}\right],
\end{aligned}
$$

where $\{A, B\}$ denotes an anticommutator, while $\Gamma_{12}$ denotes exciting process from $|1\rangle$ to $|2\rangle$ and $\Gamma_{21}$ denotes decay process from $|2\rangle$ to $|1\rangle$. 
The dephasing process (with rate $\gamma_{j}$ ) that destroys the coherence can be described by,

$$
L_{\gamma}(\rho(t))=\sum_{j=1,2} \gamma_{j}\left[\sigma_{j}^{+} \sigma_{j}^{-} \rho(t) \sigma_{j}^{+} \sigma_{j}^{-}-\frac{1}{2}\left\{\rho(t), \sigma_{j}^{+} \sigma_{j}^{-}\right\}\right] \cdot(6)
$$

Here, we treat pure dephasing in a way equivalent to the well-known Haken-Strobl model, in which pure dephasing is considered phenomenologically in terms of a classical fluctuating field [14, 32].

Additionally, two processes that lead to irreversible loss of energy are the dissipation and the sink. These effects are characterized by Lindblad operators [14, 32]

$$
L_{\Gamma}(\rho(t))=\sum_{j=1,2} \Gamma_{j}\left[\sigma_{j}^{-} \rho(t) \sigma_{j}^{+}-\frac{1}{2}\left\{\rho(t), \sigma_{j}^{+} \sigma_{j}^{-}\right\}\right]
$$

and

$$
\begin{aligned}
L_{s}(\rho(t))= & \Gamma_{s}\left[\sigma_{3}^{+} \sigma_{2}^{-} \rho(t) \sigma_{2}^{+} \sigma_{3}^{-}\right. \\
& \left.-\frac{1}{2}\left\{\rho(t), \sigma_{2}^{+} \sigma_{3}^{-} \sigma_{3}^{+} \sigma_{2}^{-}\right\}\right],
\end{aligned}
$$

where $\Gamma_{j}$ and $\Gamma_{s}$ denote the rates of dissipation and trapping, respectively. In Ref. [22, 32, 37, 43], these two processes are taken into account by adding a non-Hermitian Hamiltonians $H_{\text {loss }}=-i / 2 \sum_{j=1,2, s} \Gamma_{j}|j\rangle\langle j|$ to the system Hamiltonian (11), which is equivalent to our treatment when the sandwich term can be ignored.

The efficiency $P$ of EET is quantified by the population transferred to the sink $|3\rangle$ from the site $|2\rangle$

$$
P=\rho_{33}(T)=\Gamma_{s} \int_{0}^{T} \rho_{22}(t) d t
$$

which will be used as a measure of the transport efficiency. Thus, the question we focus on is as follows: In a given time $T$, what is the probability transferred from site $|1\rangle$ to the sink $|3\rangle$ and how the spatial structure of the system and the surrounding environment affect this transfer.

To study how the coherent evolution and the noiseinduced decoherence processes work together to reach efficient EET and illustrate the role of the phase factor in the coupling constant, we adopt a uniform and nonuniform two-level FCN to help us identify some mechanisms that underlie the energy transport [12, 18]. A uniform FCN means, $\varepsilon_{1}=\varepsilon_{2}=\varepsilon, \Gamma_{1}=\Gamma_{2}=\Gamma$ and $\gamma_{1}=\gamma_{2}=\gamma$. Given that the exciton is initially in site $|1\rangle$, then an exact analytical solution of $P$ (see the Appendix) for the case of $T=\infty$ can be obtained

$$
P=\frac{\Gamma_{s}}{\left(2 \Gamma+\Gamma_{s}\right)+\frac{A \Gamma\left(\Gamma+\Gamma_{s}+\Gamma_{21}-\Gamma_{12}\right)}{\left(4 J^{2}+A \Gamma_{12}\right)}}
$$

where $A=2 \gamma+2 \Gamma+\Gamma_{s}+\Gamma_{12}+\Gamma_{21}$. For the case of a non-uniform FCN, $\varepsilon_{1} \neq \varepsilon_{2}$ while $\gamma_{j}$ and $\Gamma_{j}$ are the same on every site. With those notations, the transfer efficiency is given by,

$$
P=\frac{\Gamma_{s}}{\left(2 \Gamma+\Gamma_{s}\right)+\frac{\Gamma\left(A^{2}+16 \Delta^{2}\right)\left(\Gamma+\Gamma_{s}+\Gamma_{21}-\Gamma_{12}\right)}{\left(4 J^{2} A+\Gamma_{12}\left(A^{2}+16 \Delta^{2}\right)\right)}},
$$

where the on-site energy gap is defined by $\Delta=$ $\left(\varepsilon_{2}-\varepsilon_{1}\right) / 2$. It is notable that, although the phase factor $\varphi$ exists in the system Hamiltonian of Eq. (11), the final efficiency $P$ does not depend on $\varphi$, which is in agreement with the findings in Ref. [15]. This is not the case, however, if the exciton is initially in a superposition of $|1\rangle$ and $|2\rangle$, as we show below.

The dephasing process (represented by the term with $\gamma_{j}$ ) does not assist the EET as expected, see Eq. (10). It is not the case, however, when we consider a non-uniform network, see Eq. (11), in this case $P$ is not a monotonic function of $\gamma$. We also observe from Fig. 2 (a) that, dephasing facilitates the transition only when $\Delta$ is larger than a certain value. The physics behind this difference is as follows. The range of on-site energies of $|1\rangle$ and $|2\rangle$ are broadened owing to the dephasing, which leads to the overlap of the two sites in energy. If the dephasing rate continues to increase, the effect of resonant mode decreases as the energy of each site is distributed over a very large interval. When $\Delta$ is quite small, or even $\Delta=0$ (uniform case), resonant mode is available without the presence of broadened site energy.

From Eq. (10) or Eq. (11), it is easy to conclude that the dissipation rate $\Gamma_{j}$ simply decreases the efficiency $P$ (see Fig. 2 (b)). The dissipation process leads to irreversible loss of energy via site $j$ to the environment which obviously against the increasing of the EET efficiency.

The influence of dephasing and dissipation in absence of relaxation (i.e., $\Gamma_{12}=\Gamma_{21}=0$ ) on the transfer efficiency in a similar network has been explored extensively in the previous works [14 17, 22]. In the following, we are interested in new results obtained when the relaxation process (usually ignored in Haken-Strobl model) ([1417, 22]) is taken into account. We find from Fig. 2 (c) that $P$ monotonically increases with $\Gamma_{12}$, but decreases with $\Gamma_{21}$, this finding can also be obtained from Eq. (10) or Eq. (11) by taking the derivative of $P$ with respect to $\Gamma_{12}$ or $\Gamma_{21}$. The observations can be understood as follows. Relaxation process represents thermal equilibration of the exciton. The diffusion of the exciton from $|1\rangle$ to $|2\rangle$ obviously increases the population of site $|2\rangle$. If $J=0$ is set, the efficiency $P=0[\mathbf{1 4}]$, since no process except coherent transfer (with coupling constant $J$ ) transfers excitons from $|1\rangle$ to $|2\rangle$. When consider the relaxation process, the situation changes. For $J=0$, Eq. (10) becomes

$$
P=\frac{\Gamma_{12} \Gamma_{s}}{\Gamma^{2}+\Gamma_{21} \Gamma_{s}+\Gamma\left(\Gamma_{12}+\Gamma_{21}+\Gamma_{s}\right)} .
$$

This suggests that excitons may be transferred to the sink, even though coherent transfer character- 
ized by $J$ is zero. As the excitons can be transferred via relaxation process, coherent transport and relaxation process work together to get a highly EET efficiency.

Fig. 2 (d) shows the dependence of the transfer efficiency $P$ on the trapping rate $\Gamma_{s}$ and how these two processes work together to reach efficient EET. In the dashed, dotted and solid lines, the relaxation rates are the same, so the improvement in the efficiency is attributed to the coherent transfer. The red-dot-dashed and black-solid lines share the same values of $J$, but the relaxation rates are different. The improvement of the efficiency is attributed to the relaxation process. Note that the thick orange-dashed and thin orange-dashed curves rise monotonically, which were not predicted in previous works [14-17]. The other curves ascend first and descend then. This is due to the relaxation ignored in the literature. This can be explained by analyzing Eq. (10). Taking derivative of $P$ with respect to $\Gamma_{s}$ (see Apendix B), we observe that, for a fixed $\Gamma$, $\Gamma_{12}, \Gamma_{21}$ and $J$, when $J^{2}<\Gamma_{12}\left(\Gamma+\Gamma_{12}+\Gamma_{21}\right), P$ is a monotonic function of $\Gamma_{s}$, it takes a maximal value $P=\frac{\Gamma_{12}}{\Gamma_{12}+\Gamma}$ with $\Gamma_{s} \rightarrow \infty$, this is shown by the thick orange-dashed and thin orange-dashed curves in Fig. 2 (d). In this case, the relaxation dominates the transfer. When the trapping rate $\Gamma_{s}$ is very small, site $|2\rangle$ couples weakly to the sink $|3\rangle$, excitations can rarely reach the sink in this situation, so the efficiency is very low. When $\Gamma_{s}$ is very large, the efficiency can be improved significantly. The highest efficiency is determined by the contribution of incoherence process given by $P=\frac{\Gamma_{12}}{\Gamma_{12}+\Gamma}$. When $J^{2}>\Gamma_{12}\left(\Gamma+\Gamma_{12}+\Gamma_{21}\right)$, $P$ is not a monotonic function of $\Gamma_{s}$ and it also takes $P=\frac{\Gamma_{12}}{\Gamma_{12}+\Gamma}$ with $\Gamma_{s} \rightarrow \infty$, but there exists an optimized value of $\Gamma_{s}$ (Fig. 2 (d)), leading to a efficiency larger than $\frac{\Gamma_{12}}{\Gamma_{12}+\Gamma}$. From Fig. 2 (d), the improvement of efficiency comes from coherent transfer. In this case, the coherent transfer dominates the transfer. When $\Gamma_{s}$ is very small, the efficiency is also very low. When $\Gamma_{s}$ increases, $P$ increases gradually to a maximum where the contribution from coherent transfer is brought into full play and excitation transferred from $|1\rangle$ to $|2\rangle$ matches the transition from $|2\rangle$ to $|3\rangle$ perfectly. Further increasing of $\Gamma_{s}$ deteriorates the coherent transfer, and eventually the efficiency decreases to the relaxation-dominated case, i.e., $P=\frac{\Gamma_{12}}{\Gamma_{12}+\Gamma}$ when $\Gamma_{s} \rightarrow \infty$. Thus, excitation transferred from $|1\rangle$ to $|2\rangle$ mismatches the transition from $|2\rangle$ to $|3\rangle$. In Ref [14], only the latter case, i.e., $J^{2}>\Gamma_{12}\left(\Gamma+\Gamma_{12}+\Gamma_{21}\right)$, is discussed because the relaxation process is not considered, i.e., $\Gamma_{12}=\Gamma_{21}=0$.

The enhancement achieved by the dephasing can be understood as the fluctuation-induced-broadening of en- ergy levels, and this gives rise to a conjecture that the fluctuations in the site energies and couplings may affect the transfer efficiency. We will focus on this question in the following discussions.

We add two energy fluctuations $\delta_{1}, \delta_{2}$, to the on-site energy gap $\Delta$ and inter-site coupling $J$ separately. The expression of $P$ Eq. (11) can be easily derived,

$$
P=\frac{\Gamma_{s}}{\left(2 \Gamma+\Gamma_{s}\right)+\frac{\Gamma\left(A^{2}+16\left(\Delta+\delta_{1}\right)^{2}\right)\left(\Gamma+\Gamma_{s}\right)}{\left(4\left(J+\delta_{2}\right)^{2} A+\Gamma_{\kappa}\left(A^{2}+16\left(\Delta+\delta_{1}\right)^{2}\right)\right)}},
$$

where $A=2 \gamma+2 \Gamma+2 \Gamma_{s}+2 \Gamma_{\kappa}$ and $\Gamma_{12}=\Gamma_{21}=\Gamma_{\kappa}$ are performed. Obviously, it monotonically increases with $\delta_{2}($ or $J)$, whereas it decreases with $\delta_{1}$ (or $\left.\Delta\right)$. This is easy to be understood. The energy gap $\Delta$ blocks the energy transfer, while the inter-site coupling $J$ that represents the overlap of sites $|1\rangle$ and $|2\rangle$ favors the transport. Fig. [3 (a) shows this result.

Now we examine the effect of phase $\varphi$ on the transfer efficiency. $\varphi$ in the coupling is determined by the spatial distribution of $|1\rangle$ and $|2\rangle$, this suggests to consider a superposition of $|1\rangle$ and $|2\rangle$ as the initial state. For the FMO complex, the spatial and temporal relaxation of exciton shows that site 1 and 6 were populated initially [29] in sunlight. In laboratory, Ref [9] reported the initial state preparation of the system in a coherent superposition of the antenna proteins electronic vibrational eigenstates with the femtosecond laser pulse (25-fs duration). Then it is reasonable to study how the initial states affect the excitation transfer efficiency. In the following, we shall shed light on this question and analyze whether the initial states have effects on the EET efficiency and whether the phase $\varphi$ can enhance the transfer. We consider two different initial states. One is a superposition state of sites $|1\rangle$ and $|2\rangle$, and the other is a classical mixture of $|1\rangle$ and $|2\rangle$.

We illustrate numerical results of the transfer efficiency as a function of $\alpha$ and $\beta$, which characterize the pure initial state of the system through $|\psi(t=0)\rangle=\cos \alpha|1\rangle+$ $\sin \alpha \exp (i \beta)|2\rangle$, where $\alpha$ denotes the population ratio between sites $|1\rangle$ and $|2\rangle$, and $\beta$ characterizes the relative phase. From Fig. 3 (c), we observe that for excitation initially excited in a superposition of sites $|1\rangle$ and $|2\rangle$, both the population ratio and the relative phase affect the energy transfer, and suitable values of $\alpha$ and $\beta$ facilitate the efficiency. The efficiency $P$ reaches its maximal value when $\alpha$ approximately equals to $\frac{\pi}{2}$ and $\frac{3 \pi}{2}$. This tells that occupation on site $|2\rangle$ helps the EET efficiency, see Eq. (9). Fig. 3 (d) shows the dependence of $P$ on $\varphi$ and $\alpha$, and Fig. 3 (e) shows the dependence of $P$ on $\varphi$ and $\beta$. These two figures indicate that the phase $\varphi$ does have effect on the efficiency when the initial state is a superposition of the two sites. This observation can be understood by an analytical solution

$$
\begin{aligned}
& P= \\
& \frac{\Gamma_{s}\left(8 J^{2}+2 \Gamma_{12} A+\Gamma A \sin ^{2} \alpha-2 J \Gamma \sin 2 \alpha \sin (\beta-\varphi)\right)(14)}{\left(4 J^{2}\left(2 \Gamma+\Gamma_{s}\right)+A \Gamma\left(\Gamma+\Gamma_{s}+\Gamma_{21}-\Gamma_{12}\right)\right)}
\end{aligned}
$$




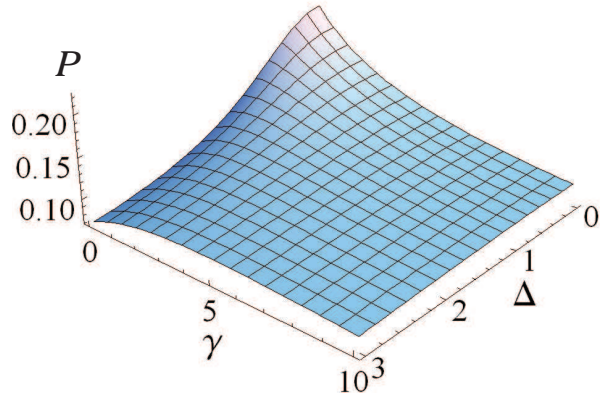

(a)

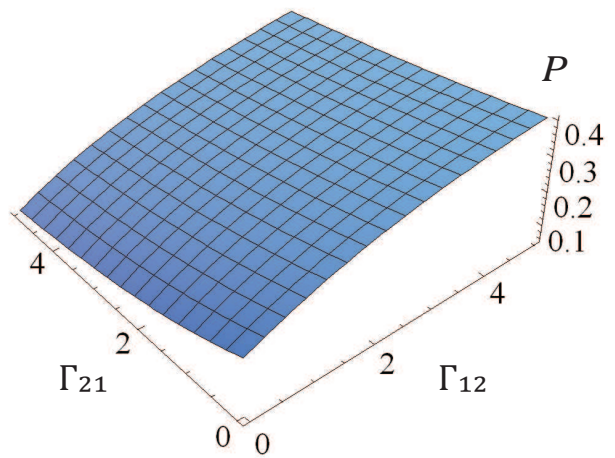

(c)

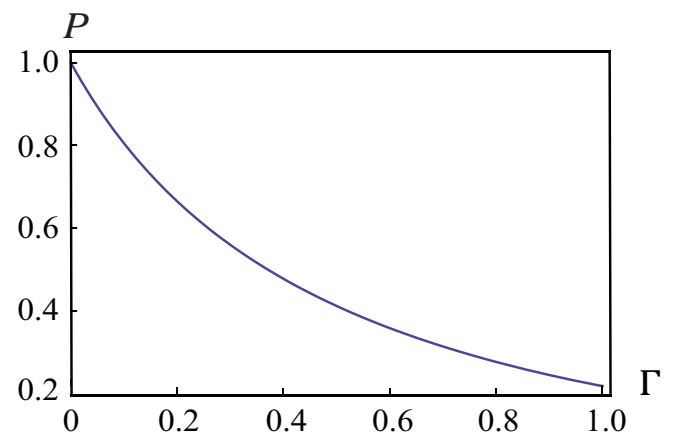

(b)

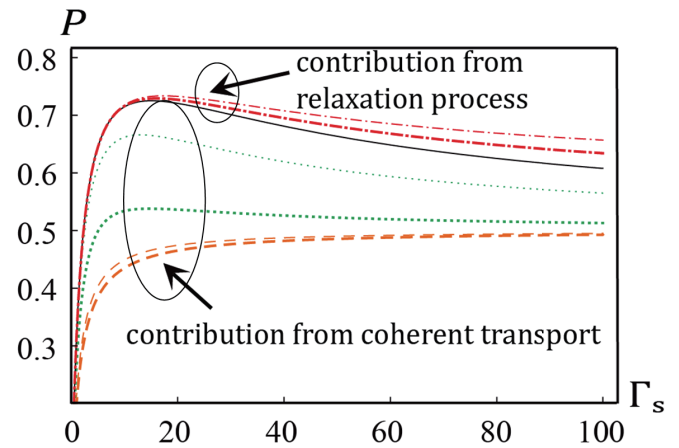

(d)

FIG. 2: (Color online) The transfer efficiency $P$. (a) $P$ vs $\gamma$ and $\Delta$. (b) $P$ vs $\Gamma$. (c) $P$ vs $\Gamma_{12}$ and $\Gamma_{21}$. (d) $P$ vs $\Gamma_{s}$. Unless otherwise noted, the other parameters chosen are $\Gamma=1, \gamma=0.1, J=1, \Gamma_{s}=1, \Gamma_{12}=\Gamma_{21}=1, \Delta=0$. In (d), the parameters is as follows: $J=0, \Gamma_{12}=\Gamma_{21}=1$ for thick orange-dashed, $J=0.5, \Gamma_{12}=\Gamma_{21}=1$ for thin orange-dashed, $J=1.5$, $\Gamma_{12}=\Gamma_{21}=1$ for thick green-dotted, $J=3, \Gamma_{12}=\Gamma_{21}=1$ for thin green-dotted, $J=4, \Gamma_{12}=\Gamma_{21}=1$ for black-solid, $J=4$, $\Gamma_{12}=\Gamma_{21}=1.2$ for thick red-dot-dashed, $\Gamma_{12}=\Gamma_{21}=1.4$ for thin red-dot-dashed.

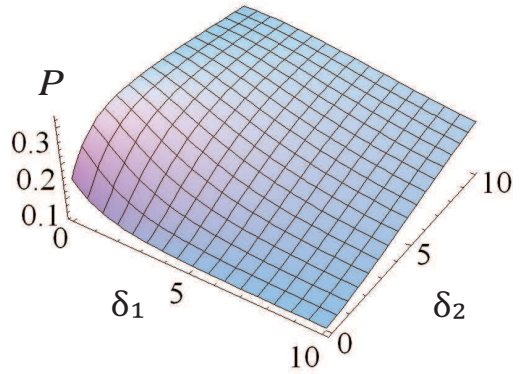

(a)

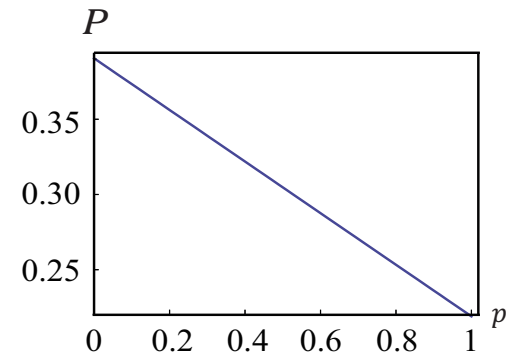

(b)

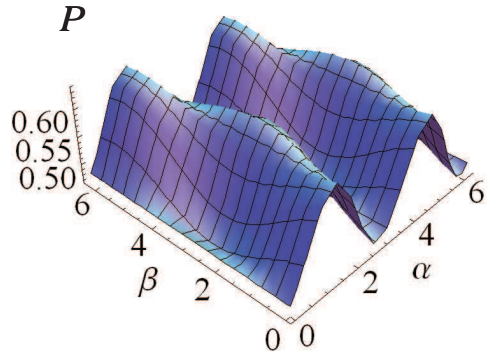

(c)

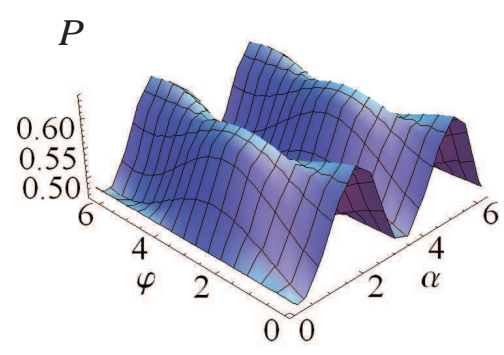

(d)

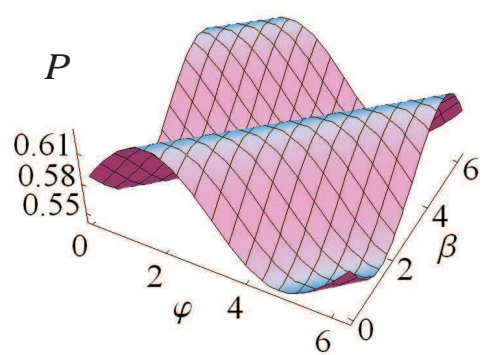

(e)

FIG. 3: (Color online) The transfer efficiency $P$. (a) $P$ vs $\delta_{1}$ and $\delta_{2}$. (b) $P$ vs $p$. (c) $P$ vs $\alpha$ and $\beta$ ( $\varphi=\frac{\pi}{3}$ ). (d) $P$ vs $\alpha$ and $\varphi\left(\beta=\frac{\pi}{3}\right)$. (e) $P$ vs $\beta$ and $\varphi\left(\alpha=\frac{\pi}{3}\right)$. Unless otherwise addressed, the other parameters chosen are $\Gamma=1, \gamma=0.1, J=1$, $\Gamma_{s}=1, \Gamma_{12}=\Gamma_{21}=1, \Delta=0$. 
obtained from Eq. (4) with $\Delta=0$ and $A=2 \gamma+$ $2 \Gamma+\Gamma_{s}+\Gamma_{12}+\Gamma_{21}$. The factor $\sin (\beta-\varphi)$ in the numerator indicates that varying the values of $\varphi$ is equivalent to varying the values of the relative phase $\beta$. This is actually equivalent to choose different coherent superposition of sites $|1\rangle$ and $|2\rangle$ as the initial states, for example,

$$
\rho(t=0)=\left(\begin{array}{cc}
\cos ^{2} \alpha & \sin \alpha \cos \alpha e^{-i \beta} \\
\sin \alpha \cos \alpha e^{i \beta} & \sin ^{2} \alpha
\end{array}\right) .
$$

Therefore, when the network is initially in a superposition state, the effect of $\varphi$ on the EET is obvious. When $\varphi$ takes a fixed value, the relative phase $\beta$ also affects the coherence of exciton transfer and the transfer efficiency $P$. But when the exciton is initially in site $|1\rangle$, neither $\varphi$ nor $\beta$ has effect.

In Ref. [47], the concepts of topological phases is introduced to explore the exciton transport in porphyrin thin films. This is the first work that addresses the joint effects of both magnetic field and coherence in molecular exciton transport. The magnetic field serves to break time-reversal symmetry and results in lattice fluxes that mimic the Aharonov-Bohm phase acquired by electrons. The first blueprint for realizing topological phases of matter in molecular aggregates stimulates us to study the EET dynamics from new point of view.

Another type of initial states we consider is mixed state with mixing rate $p, p|1\rangle\langle 1|+(1-p)| 2\rangle\langle 2|$, see Fig. 3(b). We observe that $p$ works like $\alpha$ denoting the population weight. More population on site $|2\rangle$ favors the transport, and $\varphi$ has no effect on the efficiency. This is because for sites $|1\rangle$ and $|2\rangle$ being initially in a classical mixed state, there is no coherence initially, leading to no influence of $\varphi$ on the efficiency. Actually, we obtain an exact analytical expression for $P$ in this case,

$$
P=\frac{\Gamma_{s}}{\left(2 \Gamma+\Gamma_{s}\right)+\frac{A \Gamma\left[(2 p-1) \Gamma+p \Gamma_{s}\right]}{A\left[(1-p) \Gamma+\Gamma_{\kappa}\right]+4 J^{2}}},
$$

where $A=2 \gamma+2 \Gamma+2 \Gamma_{s}+2 \Gamma_{\kappa}$ and $\Gamma_{12}=\Gamma_{21}=\Gamma_{\kappa}$ are performed. From this expression we easily know that $P$ is independent of $\varphi$.

The effect of phase $\varphi$ on the EET dynamics and efficiency will be discussed further in section V, using a time-convolutionless master equation.

\section{EET DYNAMICS IN NON-MARKOVIAN CASE}

In section III, we treat pure dephasing in a way equivalent to the Haken-Strobl model, in which the environment is modeled as a spatially uncorrelated classical white noise. Now we consider the EET dynamics using the Lindblad master equation derived from a time-convolutionless master equation within the weakcoupling, Born-Markovian and secular approximations [32, 48, 49]. In contrast to the Haken-Strobl model, the environment is treated in a quantum way,

$$
\dot{\rho}(t)=-i\left[H_{S}, \rho\right]+L_{\Gamma}(\rho(t))+L_{s}(\rho(t))+L(\rho(t)),
$$

where the first three terms on the right side are the same as those in Eq. (4), representing coherent evolution, dissipation and sink, respectively. $L(\rho(t))$ is the Lindblad superoperator given by [35, 49]:

$$
\begin{aligned}
& L(\rho(t))=\sum_{m, \omega} \gamma(\omega, t) \\
& {\left[A_{m}(\omega) \rho(t) A_{m}^{\dagger}(\omega)-\frac{1}{2}\left\{A_{m}^{\dagger}(\omega) A_{m}(\omega), \rho(t)\right\}\right],}
\end{aligned}
$$

where $\gamma(\omega, t)$ is the non-Markovian decoherence rate under the assumption of Ohmic spectral density with exponential cutoff

$$
J(\omega)=\frac{\lambda}{\omega_{c}} \omega \exp \left(-\frac{\omega}{\omega_{c}}\right),
$$

where $\omega_{c}$ is the cutoff frequency and $\lambda$ is the strength of the system-bath coupling. Generally, the non-Markovian decoherence rate is time-dependent given by

$$
\begin{aligned}
& \gamma(\omega, t)=2 \int_{0}^{\infty} d \tilde{\omega} J(\tilde{\omega}) \\
& \left(n(\tilde{\omega}) \frac{\sin [(\omega+\tilde{\omega}) t]}{\omega+\tilde{\omega}}+[n(\tilde{\omega})+1] \frac{\sin [(\omega-\tilde{\omega}) t]}{\omega-\tilde{\omega}}\right)
\end{aligned}
$$

where $n(\omega)=\left[\exp \left(\omega / k_{B} T\right)-1\right]^{-1}$ is the bosonic distribution. In the Markovian limit $(t \rightarrow \infty)$, the decoherence rate can be obtained

$$
\gamma(\omega, \infty)=2 \pi J(|\omega|)|n(-\omega)| .
$$

The dephasing rate can be derived from Eq. (20) in the limit $\omega \rightarrow 0$

$$
\gamma_{\varphi}(t)=2 \int_{0}^{\infty} d \tilde{\omega} J(\tilde{\omega}) \operatorname{coth}\left(\frac{\tilde{\omega}}{2 k_{B} T}\right) \frac{\sin (\tilde{\omega} t)}{\tilde{\omega}} .
$$

In the Markovian limit $(t \rightarrow \infty)$, the dephasing rate becomes

$$
\gamma_{\varphi}(\infty)=\frac{2 \pi k_{B} T \lambda}{\omega_{c}}
$$

In the later simulation, unless otherwise noticed, we set $\lambda=50 \mathrm{~cm}^{-1}, \omega_{c}=50 \mathrm{~cm}^{-1}$ as in Ref. [35], and note that in units of $\hbar=1$, we have $1 \mathrm{ps}^{-1}=5.3 \mathrm{~cm}^{-1}$.

$A_{m}(\omega)$ is the jump operators defined by

$$
A_{m}(\omega)=\sum_{\lambda_{k}-\lambda_{l}=\omega} c_{m}^{*}(k) c_{m}(l)\left|\lambda_{k}\right\rangle\left\langle\lambda_{l}\right| .
$$

The sum runs over all possible transitions in the single exciton manifold. In our model, the basis $\left|\lambda_{k}\right\rangle=$ 
$\sum_{m=1,2} c_{m}(k)|m\rangle(k=1,2)$ is composed by the eigenbasis of Hamiltonian (1), $H_{S}\left|\lambda_{k}\right\rangle=\lambda_{k}\left|\lambda_{k}\right\rangle$. Simple algebra yields,

$$
\begin{aligned}
& \lambda_{1}=\frac{1}{2}\left[\varepsilon_{1}+\varepsilon_{2}+\sqrt{\left(\varepsilon_{1}-\varepsilon_{2}\right)^{2}+4 J^{2}}\right] \\
& \lambda_{2}=\frac{1}{2}\left[\varepsilon_{1}+\varepsilon_{2}-\sqrt{\left(\varepsilon_{1}-\varepsilon_{2}\right)^{2}+4 J^{2}}\right] .
\end{aligned}
$$

And the corresponding eigenstates are,

$$
\begin{aligned}
& \left|\lambda_{1}\right\rangle=\sin \theta|1\rangle+\cos \theta \exp (i \varphi)|2\rangle, \\
& \left|\lambda_{2}\right\rangle=\cos \theta|1\rangle-\sin \theta \exp (i \varphi)|2\rangle .
\end{aligned}
$$

where the mixing angle is given by

$$
\begin{aligned}
& \sin \theta=\frac{\varepsilon_{1}-\varepsilon_{2}+\sqrt{4 J^{2}+\left(\varepsilon_{1}-\varepsilon_{2}\right)^{2}}}{\sqrt{4 J^{2}+\left(\varepsilon_{1}-\varepsilon_{2}+\sqrt{4 J^{2}+\left(\varepsilon_{1}-\varepsilon_{2}\right)^{2}}\right)^{2}}}, \\
& \cos \theta=\frac{2 J}{\sqrt{4 J^{2}+\left(\varepsilon_{1}-\varepsilon_{2}+\sqrt{4 J^{2}+\left(\varepsilon_{1}-\varepsilon_{2}\right)^{2}}\right)^{2}}} .
\end{aligned}
$$

Thus the jump operators for relaxation are given by

$$
\begin{aligned}
& A_{1,2}\left(\omega_{12}\right)= \pm \sin \theta \cos \theta\left|\lambda_{1}\right\rangle\left\langle\lambda_{2}\right|, \\
& A_{1,2}\left(\omega_{21}\right)= \pm \sin \theta \cos \theta\left|\lambda_{2}\right\rangle\left\langle\lambda_{1}\right|,
\end{aligned}
$$

for dephasing, they are

$$
\begin{aligned}
& A_{1}(0)=\sin ^{2} \theta\left|\lambda_{1}\right\rangle\left\langle\lambda_{1}\left|+\cos ^{2} \theta\right| \lambda_{2}\right\rangle\left\langle\lambda_{2}\right|, \\
& A_{2}(0)=\cos ^{2} \theta\left|\lambda_{1}\right\rangle\left\langle\lambda_{1}\left|+\sin ^{2} \theta\right| \lambda_{2}\right\rangle\left\langle\lambda_{2}\right| .
\end{aligned}
$$

In the Hilbert space spanned by the eigenstates of the Hamiltonian, $L(\rho(t))$ can be written as

$$
\begin{aligned}
L(\rho(t))= & \sum_{n=1,2} \gamma_{n}\left(\sigma_{n n} \rho(t) \sigma_{n n}-\frac{1}{2}\left\{\sigma_{n n}, \rho(t)\right\}\right) \\
& +\gamma_{12}\left(\sigma_{11} \rho(t) \sigma_{22}+\sigma_{22} \rho(t) \sigma_{11}\right) \\
& +\Gamma_{21}\left(\sigma_{21} \rho(t) \sigma_{12}-\frac{1}{2}\left\{\sigma_{11}, \rho(t)\right\}\right) \\
& +\Gamma_{12}\left(\sigma_{12} \rho(t) \sigma_{21}-\frac{1}{2}\left\{\sigma_{22}, \rho(t)\right\}\right),
\end{aligned}
$$

where $\sigma_{i j}=\left|\lambda_{i}\right\rangle\left\langle\lambda_{j}\right|$. The first two terms represent the dephasing and the last two terms describe relaxation, which is similar to Eq. (5) and Eq. (6). The relaxation and dephasing here is expressed in the basis composed of the Hamiltonian eigenstate, different from the phenomenological treatment in Eq. (5) and Eq. (6). The decoherence rates defined in Eq. (20-23) take,

$$
\begin{aligned}
\gamma_{n} & =\left(\sin ^{4} \theta+\cos ^{4} \theta\right) \gamma_{\varphi}(t), \\
\gamma_{12} & =2 \sin ^{2} \theta \cos ^{2} \theta \gamma_{\varphi}(t), \\
\Gamma_{12} & =2 \sin ^{2} \theta \cos ^{2} \theta \gamma\left(\omega_{12}, t\right), \\
\Gamma_{21} & =2 \sin ^{2} \theta \cos ^{2} \theta \gamma\left(\omega_{21}, t\right),
\end{aligned}
$$

for non-Markovian dynamics. They take,

$$
\begin{aligned}
\gamma_{n} & =\left(\sin ^{4} \theta+\cos ^{4} \theta\right) \gamma_{\varphi}(\infty), \\
\gamma_{12} & =2 \sin ^{2} \theta \cos ^{2} \theta \gamma_{\varphi}(\infty), \\
\Gamma_{12} & =2 \sin ^{2} \theta \cos ^{2} \theta \gamma\left(\omega_{12}, \infty\right), \\
\Gamma_{21} & =2 \sin ^{2} \theta \cos ^{2} \theta \gamma\left(\omega_{21}, \infty\right),
\end{aligned}
$$

for Markovian case, where $\omega_{k l}=\lambda_{k}-\lambda_{l}$ is the transition frequency. The parameters chosen are: $J=87 \mathrm{~cm}^{-1}$, $\varepsilon_{1}=0$ and $\varepsilon_{2}=120 \mathrm{~cm}^{-1}$, equivalent to the Hamiltonian of sites $|1\rangle$ and $|2\rangle$ subsystem in the FMO complex in Ref. [13].

With these arrangements, we numerically calculated the time dependence of the decoherence rates, the time evolution of the population on the three sites and the coherence element of the density matrix in the site basis, i.e., $\rho_{m n}(t)=\langle m|\rho(t)| n\rangle$. Markovian and non-Markovian decoherence rates and transfer dynamics given by Eqs. (20- 23) are plotted in Fig. 4 and Fig. 5 . The non-Markovian relaxation rates oscillate, taking positive and negative values, and finally converge to values in the Markovian limit [35]. The dephasing rates in the nonMarkovian case start with zero and similarly, converge to that in the Markovian limit. We also observe that higher temperature leads to larger amplitude of oscillation in the relaxation rates. For the dynamics, the oscillations of population in sites $|1\rangle$ and $|2\rangle$ and coherence element in the Markovian case at low temperatures are almost the same with those in the non-Markovian case. At higher temperatures, however, considerable differences can be observed from Fig. 4 and Fig. 5 , Oscillations in the population and coherence element last shortly, whereas in the non-Markovian case, they last long. This can be explained as follows. For Markovian case, the dephasing rate is linearly proportional to the temperature according to Eq. (23), whereas at the same temperature, non-Markovian dephasing rate is much smaller at short time from Fig. 4. In addition, the Markovian relaxation rate is always positive, while for the non-Markovian case, the relaxation rate may take positive and negative. Since the relaxation process represents the diffusion of excitons through the system, oscillatory relaxation rates of nonMarkovian dynamics lead to beatings of the population and coherence element, which reflects the memory effect of the non-Markovian environment.

These factors lead to the difference of the population on site $|3\rangle$ between Markovian and nonMarkovian cases. Note that we consider the population $\rho_{33}$ accumulated on the site 3 for a time of $t=1 \mathrm{ps}$, i.e., we choose $\rho_{33}(1 \mathrm{ps})$ to quantify the transfer efficiency, this is different from the case in Sec. III, where we use $\rho_{33}(\infty)$. Similarly, at low temperatures, the population in site $|3\rangle$ in Markovian case is almost the same as in non-Markovian case. At higher temperatures, it exhibits a slight difference (see Fig. 4 and Fig. 6] (a)), which can also be explained by the different behavior of decoherence rates between Markovian and non- 

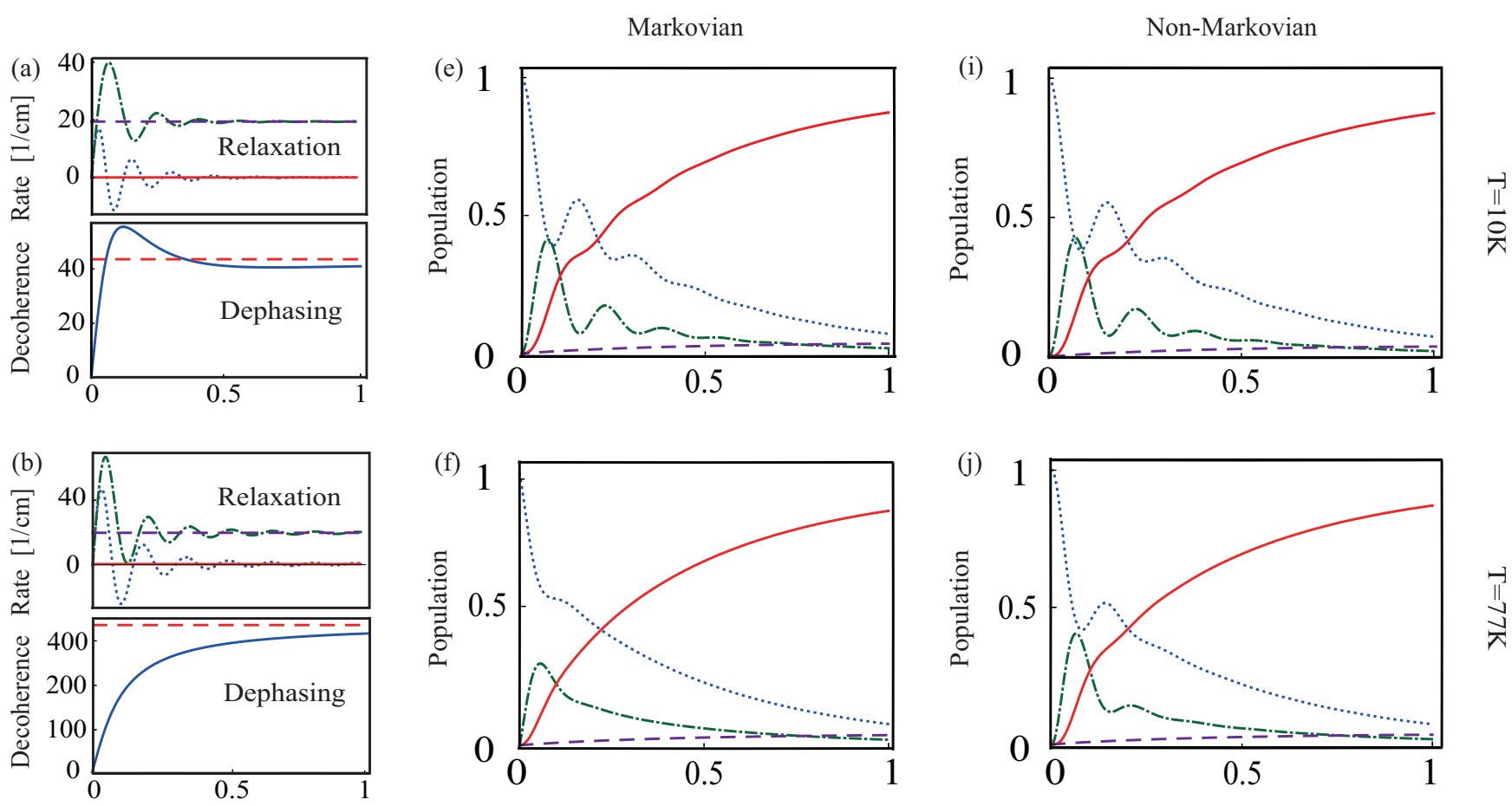

$\stackrel{\mathbb{\pi}}{\underset{\pi}{*}}$
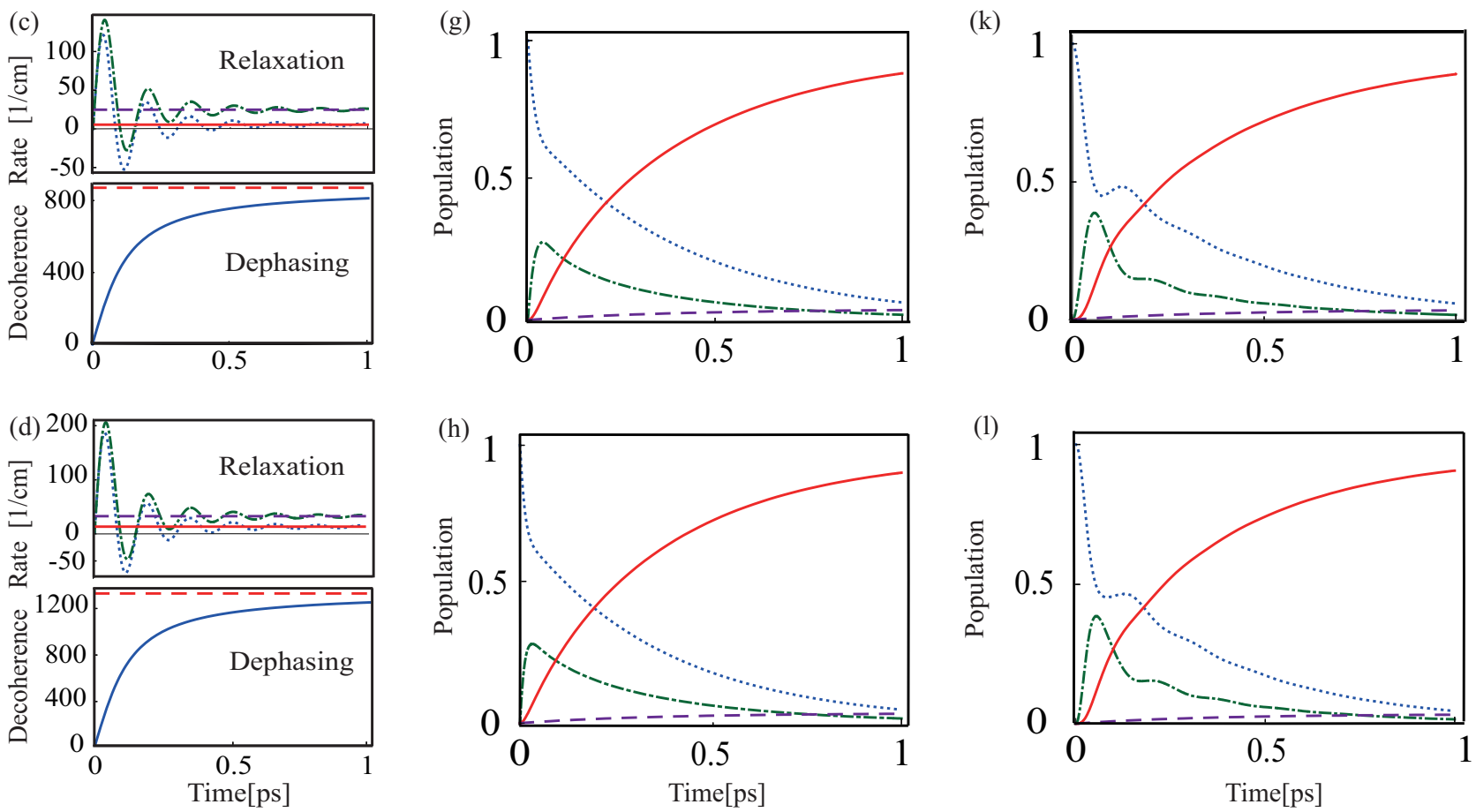

FIG. 4: (Color online) Time-dependent decoherence rates from Eq. 201212223) and time evolution of population on each site from Eq.(17) for Markovian and non-Markovian dynamics. The left panels (Fig. 4 (a) - (d)) show the time evolution of relaxation (upper panel) and dephasing (lower panel) rates. The parameters in the spectral density are $\omega_{c}=50 \mathrm{~cm}{ }^{-1}$ and $\lambda=$ $50 \mathrm{~cm}^{-1}$ as used in Ref. [35]. The relaxation rates (blue-dotted for $\omega_{12}$ and green-dot-dashed for $\omega_{21}$ ) oscillate, they may take negative value and converge to values in the Markovian limit (red-solid for $\omega_{12}$, the purple-dashed line is for $\omega_{21}$ ). Similarly, the dephasing rates in the non-Markovian case (blue solid) start with zero and converge to these in the Markovian limit (reddashed). Fig. 4 (e) - (l) show the population on each site as a function of time: $\rho_{00}$ in purple-dashed, $\rho_{11}$ in blue-dotted, $\rho_{22}$ in green-dot-dashed, and $\rho_{33}$ in red-solid. (e) - (h) is plotted for the Markovian case, and (i) - (l) for the non-Markovian case. The temperatures $T$ and phase $\varphi$ change from figure to figure. The population on the reaction center $\rho_{33}$ with the same temperatures $T$ is also illustrated (red-solid for $\varphi=0$, orange-dashed for $\varphi=\pi / 2$, black dot-dashed for $\varphi=\pi$ ). The Hamiltonian is taken to be the same as that in Ref. [13]. Dissipation and trapping rates we chose are $\Gamma_{1}=0.1 \mathrm{ps}^{-1}, \Gamma_{2}=0.1$ $\mathrm{ps}^{-1}$, and $\Gamma_{s}=10 \mathrm{ps}^{-1}$. 


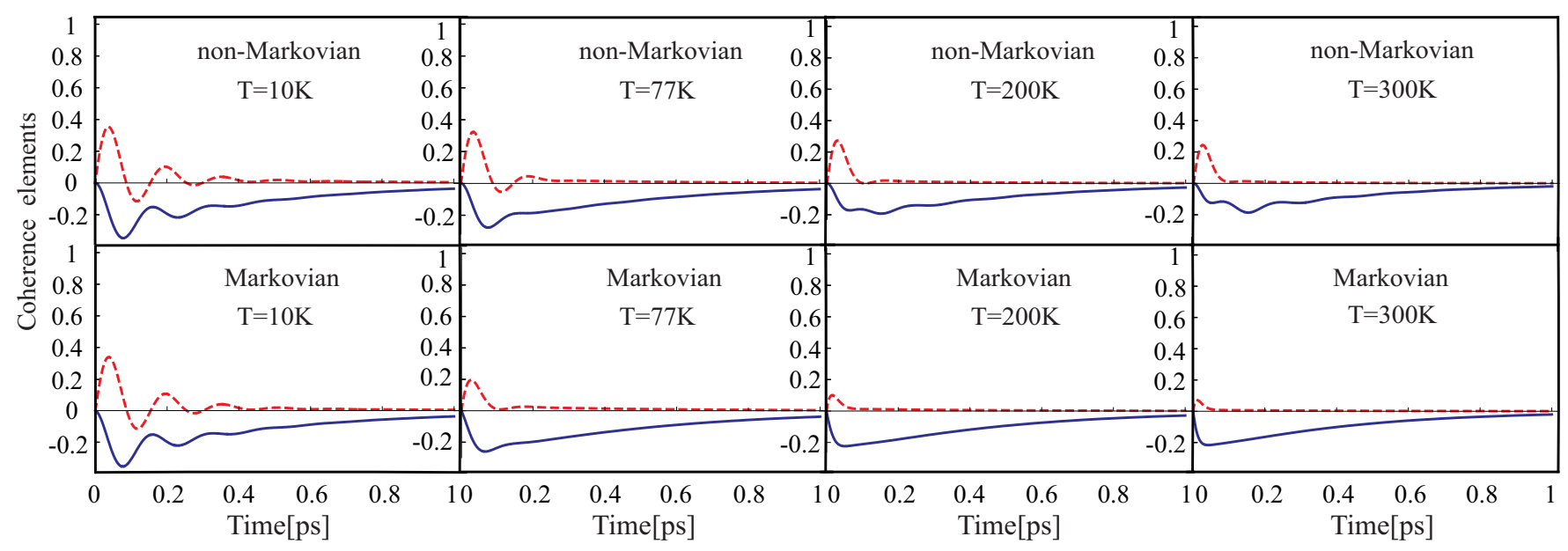

FIG. 5: (Color online) Time evolution of the coherence elements (Re $\rho_{12}$ red-dashed, Im $\rho_{12}$ blue-solid) from Eq. (17) for Markovian and non-Markovian dynamics. The excitonic Hamiltonian is taken to be the form equal to that in Ref. [13]. The parameters are $\lambda=50 \mathrm{~cm}^{-1}, \omega_{c}=50 \mathrm{~cm}^{-1}$, phase $\varphi=0$, and room temperature $T=300 \mathrm{~K}$. Dissipation and trapping rates we choose are $\Gamma_{1}=0.1 \mathrm{ps}^{-1}, \Gamma_{2}=0.1 \mathrm{ps}^{-1}$, and $\Gamma_{s}=10 \mathrm{ps}^{-1}$.

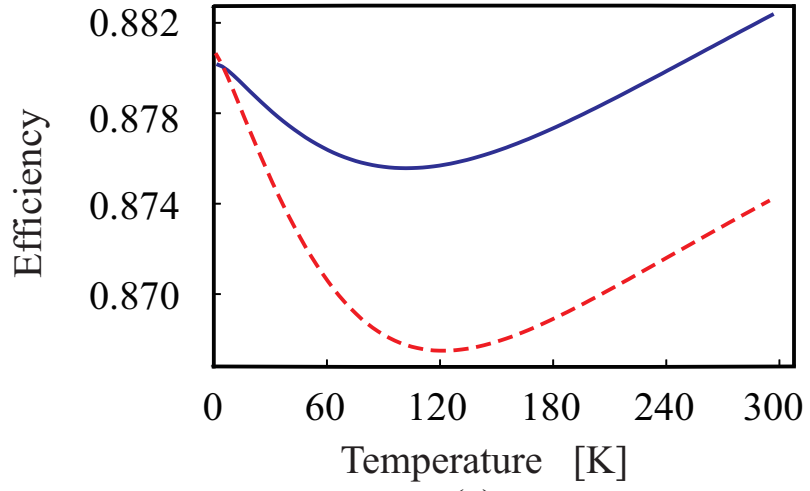

(a)

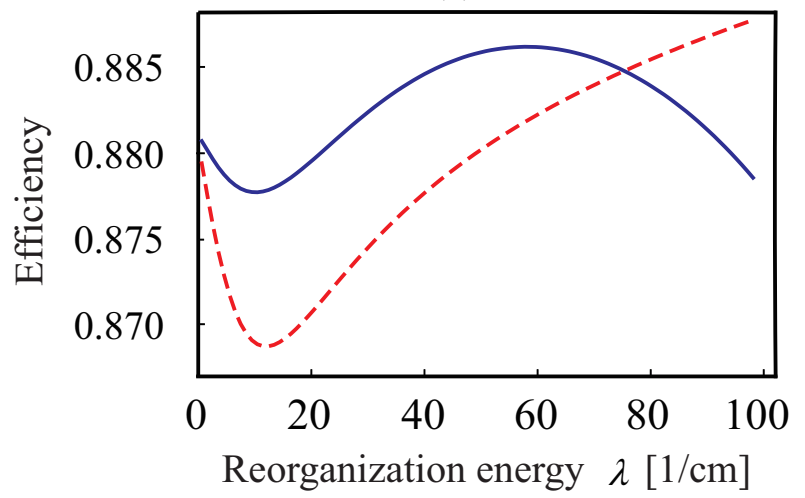

(c)

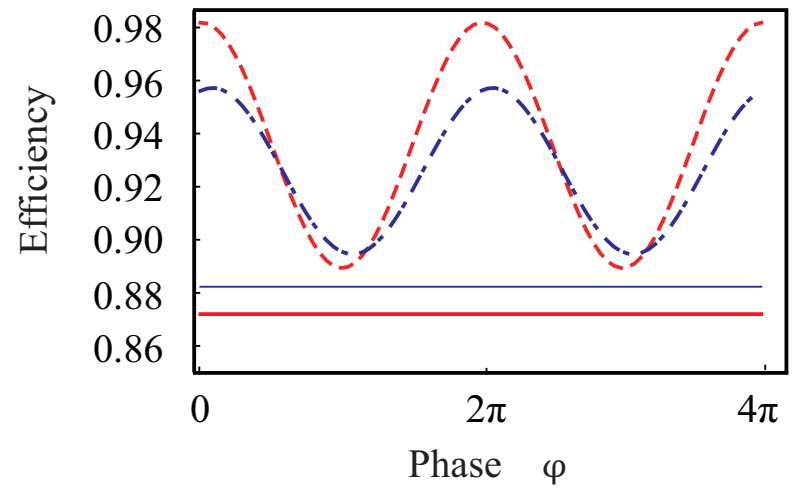

(b)

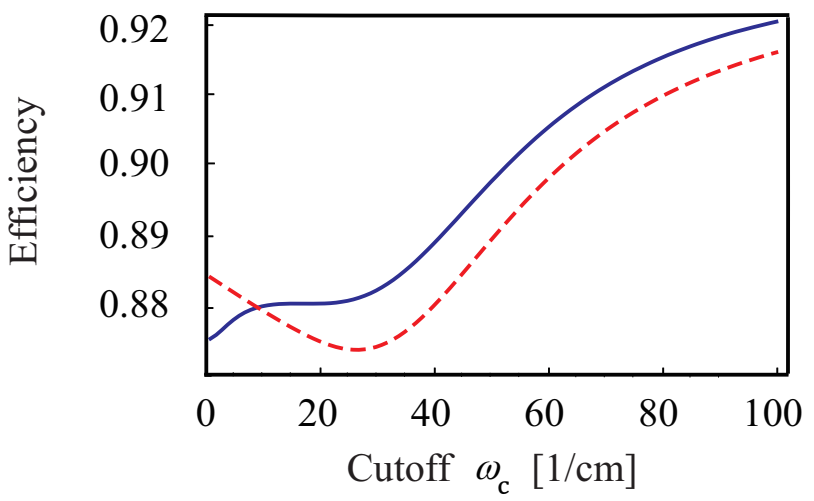

(d)

FIG. 6: (Color online) The transfer efficiency $P$ as a function of different temperatures, the phases $\varphi$ and the main decoherence parameters (reorganization energy $\lambda$, cutoff $\omega_{c}$ ) for Markovian (red-dashed) and non-Markovian (blue-soloid) cases. The parameters are $\omega_{c}=30 \mathrm{~cm}^{-1}, \lambda=30 \mathrm{~cm}^{-1}$, phase $\varphi=0$, which is typical for some natural energy transfer systems like chromophores in photosynthetic systems, and the temperature is $T=300 \mathrm{~K}$. Dissipation rate $\Gamma_{1,2}$, trapping rate $\Gamma_{s}$, and excitonic Hamiltonian takes the same values as in Fig. 4. In (b), thick red-solid for Markovian and thin blue-solid for nonMarkovian with the initial state $\rho_{11}(0)=1$, and red-dashed for Markovian and blue-dot-dashed for non-Markovian with the initial state a superposition state $|\psi(t=0)\rangle=\frac{1}{2}|1\rangle+\frac{\sqrt{3}}{2}|2\rangle$. 
Markovian cases. Note that the difference in the efficiency between Markovian and non-Markovian cases is not so large. Furthermore, from Fig. 6 (a), we find that the temperature does not change much the population on site $|3\rangle$, hence the efficiency is robust against the temperature. This is consistent with recent experimental results that the nature photosynthesis are able to transport incident light energy with nearly $100 \%$ efficiency in hot and wet conditions [2-10].

Fig. 6 (b) shows the efficiency as a function of the phase $\varphi$ for Markovian and non-Markovian cases. Fig. 6 (b) show that the phase factor $\varphi$ has influence on neither the EET dynamics nor the efficiency with initial state $\rho_{11}(0)=1$, this observation holds true for both Markovian and non-Markovian cases. When we consider a superposition of $|1\rangle$ and $|2\rangle$ as the initial state, the effect of $\varphi$ on the efficiency is clear from Fig. 6 (b), and the improvement in the efficiency compared with $\rho_{11}(0)=1$ is obvious. These results are in agreement with the conclusions in section III. The effect of the phase factor on the EET depends on initial states. The difference in the efficiency for Markovian and non-Markovian cases is very small.

In Fig. 6 (c), we show the dependence of transfer efficiency on the reorganization energy $\lambda$ of the spectral density. From Eq. (20-23), we see that relaxation and dephasing rates are both proportional to $\lambda$. As discussed above, the dephasing destroys the coherence and hinders the energy transfer when the on-site energy gap $\Delta$, i.e., $\omega_{12}\left(\omega_{21}\right)$ in this section, is sufficiently small compared with the dephasing rates, while it improves the energy transfer when $\Delta$ is suitably large compared with the dephasing rates. But the relaxation terms describing the exciting and decay processes among the eigenstates $\left|\lambda_{1}\right\rangle$ and $\left|\lambda_{2}\right\rangle$ would only accelerate the energy transfer. With $\lambda$ getting larger from a very small value, both the dephasing rates and relaxation rates become larger. Therefore, the efficiency varies nonmonotonically with $\lambda$ increasing for both Markovian and non-Markovian dynamics. The difference in the EET dynamics for Markovian and non-Markovian cases results from the different decoherence rates when $\lambda$ changes (Fig. 4 (a) (d)). The difference in the transfer efficiency can be even small, see Fig. 6 (c), this is because the transfer efficiency is defined as an accumulation of population on site 3 . It is worth addressing that the master equation (17) is obtained within the weak-coupling limit. Therefore, for small values of $\lambda$, the numerical results is reasonable. When $\lambda$ is large, the master equation (17) is theoretically not applicable, this means it can not be derived as in textbook, but it is allowed to use the master equation phenomenonaly to describe the EET.

Fig. 6 (d) shows the dependence of $P$ as a function of $\omega_{c}$. Eq. (19) tells that the spectral density $J(\omega)$ is not a monotonic function of $\omega_{c}$, and the four decoherence rates change differently with $\omega_{c}$. Similarly, when $\omega_{c}$ changes, the roles that the relaxation and dephasing play in the dynamics change for both Markovian and non-Markovian dynamics, leading to the difference in the dependence of $P$ on $\omega_{c}$ between Markovian and non-Markovian cases, see in Fig. 6 (d). Notice that a larger cutoff $\omega_{c}$ means stronger couplings between the system and environment, so the master equation (17) becomes not applicable from the respect that it can be derived by the use of week-coupling approximation.

The reason why the different surrounding environments can affect the EET dynamics but exhibit no influence on the efficiency (Fig. 4 - Fig. 6) lies in that: when the EET dynamics is in the stage of coherent oscillation, the exciton is transferred relatively faster. The surrounding environment and the temperature may influence the duration of coherent oscillation, but before this stage ends, the exciton has been transferred to the sink $|3\rangle$ with a large probability, no matter how long this period of coherent oscillation is. This suggestes that quantum coherence play a significant role in achieving the remarkable efficient EET [11 13]. The explanation above gives an intuitive picture how the exciton is transferred through the system which will be further illustrated in terms of the notion of quantumness in Sec. V.

It is notable that the efficiency of EET for Markovian and non-Markovian cases discussed in [35] exhibits considerable difference. It is due to the different measure to elucidate the EET efficiency. In this paper, we adopt trapping sites $|3\rangle$ (with trapping rate $\Gamma_{s}$ ) to model the sink while [35] ignore the trapping process and utilize another kind of measure given by

$$
P=\frac{1}{\tau} \int_{0}^{\tau} d t\langle M|\rho(t)| M\rangle,
$$

where $\tau$ is the total integration time and $|M\rangle$ is a particular site.

\section{QUANTUMNESS OF ENERGY CURRENT IN EET}

In Sec. IV, we have shown that quantum coherent wave-like oscillations of the populations in sites $|1\rangle$ and $|2\rangle$ last up to $500 \mathrm{fs}$ at temperature $T=10 \mathrm{~K}$, and become shorter with the temperature increasing for both Markovian and non-Markovian dynamics. In the following, we will quantify how quantum the EET dynamics is and study the influence of environment and spatial structure of the system on this process. As energy transfer constitutes the main function of the system, we focus on the quantumness of the energy transfer current [50]. As is widely accepted, the most classical states of a quantum system are the pointer states, which are einselected by the decoherence process. For the case of a measurement of energy current, the pointer states are the eigenstates 
of the energy current operator [45, 46]. We then can measure the distance of the density matrix to the convex set of the pointer states [44, 51, 52], i.e., classical mixing the pointer states, and define the quantumness as the minimum distance.

\section{A. Energy currents in the transport}

First, we derive the energy current operator for our system utilizing a well-developed theory by Hardy 53. which was widely used by a large amount of succeeding literatures $[54,58]$. An energy current operator $\vec{j}(x)$ can be obtained from a continuity equation

$$
\dot{H}(\mathrm{x})+\nabla \cdot \overrightarrow{\mathrm{j}}(\mathrm{x})=0
$$

with $H(\mathrm{x})$ the local energy density. For the Hamiltonian (11) in tight-binding approximation, $H=\sum_{m, n}^{2} h_{m n}|m\rangle\langle n|$, we rewrite the decomposition of the Hamiltonian in terms of local excitations as

$$
\begin{aligned}
& H=h_{1}+h_{2}, \\
& h_{m}=\frac{1}{2} \sum_{n=1}^{2}\left(h_{m n}|m\rangle\langle n|+H . c .\right),
\end{aligned}
$$

Consider a one-dimensional chain, the current can flow only in one direction, hence $\vec{j}$ has only one component, which will be denoted by $j$, and its value on site $m$ denoted by $j_{m}$. Taking as equidistant with lattice constant $a$, we obtain the discretized form of Eq. (34)

$$
\frac{\partial}{\partial t} \frac{h_{m}}{a}=\frac{j_{m}^{l}-j_{m}^{r}}{a}
$$

with $j_{m}^{l(r)}$ the energy flux at positive $x$ direction on the left (right) of site $m$. The balance of the currents $s_{1 \rightarrow 2}$ from site $|1\rangle$ to site $|2\rangle$ and $s_{2 \rightarrow 1}$ from site $|2\rangle$ to site $|1\rangle$ forms current $j_{m}^{l(r)}$. With these observations, we have

$$
\begin{aligned}
& \frac{\partial}{\partial t} h_{1}=s_{2 \rightarrow 1}-s_{1 \rightarrow 2}, \\
& \frac{\partial}{\partial t} h_{2}=s_{1 \rightarrow 2}-s_{2 \rightarrow 1} .
\end{aligned}
$$

At the same time, we calculate the left-side of Eq. (37) by means of Heisenberg's equation of motion,

$$
\frac{\partial}{\partial t} h_{m}=i\left[H, h_{m}\right]
$$

We have

$$
\begin{aligned}
\frac{\partial}{\partial t} h_{1} & =\frac{i}{2} \sum_{l}\left(h_{21} h_{1 l}|2\rangle\left\langle l\left|-h_{12} h_{2 l}\right| 1\right\rangle\langle l|-H . c .\right), \\
\frac{\partial}{\partial t} h_{2} & =\frac{i}{2} \sum_{l}\left(h_{12} h_{2 l}|1\rangle\left\langle l\left|-h_{21} h_{1 l}\right| 2\right\rangle\langle l|-H . c .\right) .
\end{aligned}
$$

Comparing with Eq. (37), we obtain the energy current

$$
\begin{aligned}
& s_{1 \rightarrow 2}=\frac{i}{2} \sum_{l}\left(h_{12} h_{2 l}|1\rangle\langle l|-H . c .\right), \\
& s_{2 \rightarrow 1}=\frac{i}{2} \sum_{l}\left(h_{21} h_{1 l}|2\rangle\langle l|-H . c .\right) .
\end{aligned}
$$

The energy current operator is

$$
\begin{aligned}
& j_{12}=s_{1 \rightarrow 2}-s_{2 \rightarrow 1} \\
& =\frac{i}{2}\left(h_{11}+h_{22}\right)\left(h_{12}|1\rangle\left\langle 2\left|-h_{21}\right| 2\right\rangle\langle 1|\right) .
\end{aligned}
$$

Therefore,

$$
\begin{aligned}
& \left\langle j_{12}(t)\right\rangle=\operatorname{tr}\left[j_{12} \rho(t)\right] \\
& =\frac{i}{2}\left(\varepsilon_{1}+\varepsilon_{2}\right) J\left(e^{-i \varphi} \rho_{21}(t)-e^{i \varphi} \rho_{12}(t)\right),
\end{aligned}
$$

where $\rho_{12}(t)$ and $\rho_{21}(t)$ are the time evolution of the coherence elements obtained from Eq. (17). We numerically calculate $\left\langle j_{12}(t)\right\rangle$ at different temperatures for both Markovian and non-Markovian dynamics, and present the results in Fig. 7. The result of $\left\langle j_{12}(t)\right\rangle$ is given in unit of $6.57 \times 10^{-4} \mathrm{eV} / \mathrm{ps}$. As phase $\varphi$ does not affect the EET dynamics given the initial state $\rho_{11}(0)=1$, we don't consider the influence of $\varphi$.

Lower temperature leads to longer oscillations and larger oscillation amplitude for both Markovian and nonMarkovian cases, and the amplitude difference between the two cases become more obvious at larger temperature. This is because the coherence die out very fast and more differences between the Markovian and nonMarkovian dynamics emerge at high temperature, which, from Eq. (42), will lead to smaller oscillations and larger amplitude difference. After the initial time, energy current becomes small but finite up to $1 \mathrm{ps}$, which helps in understanding the EET process further based on the conclusion obtained in Sec. IV as follows: energy transfer begins with quantum coherent population exchange between sites $|1\rangle,|2\rangle$ and $|3\rangle$, then small currents gradually redistribute energy between the three sites and finally the system arrives at an equilibrium.

\section{B. Quantumness}

The eigenvectors of $j_{12}$, i.e., the pointer states [45, 46], are given by

$$
\left|\nu^{1}\right\rangle=\left(\begin{array}{c}
\frac{i e^{-i \varphi}}{\sqrt{2}} \\
\frac{1}{\sqrt{2}}
\end{array}\right),\left|\nu^{2}\right\rangle=\left(\begin{array}{c}
\frac{-i e^{-i \varphi}}{\sqrt{2}} \\
\frac{1}{\sqrt{2}}
\end{array}\right)
$$

defining the relevant pure classical states for the energy transfer. The minimum distance of $\rho(t)$ to the convex set of classical states is defined as quantumness of the current $j_{12}$ [50],

$$
Q_{12}(\rho)=\min _{\left\{p_{i} \mid p_{i} \geq 0, \sum_{i} p_{i}=1\right\}} \| \rho-\sum_{l=1,2} p_{l}\left|\nu^{l}\right\rangle\left\langle\nu^{l}\right| \|,
$$



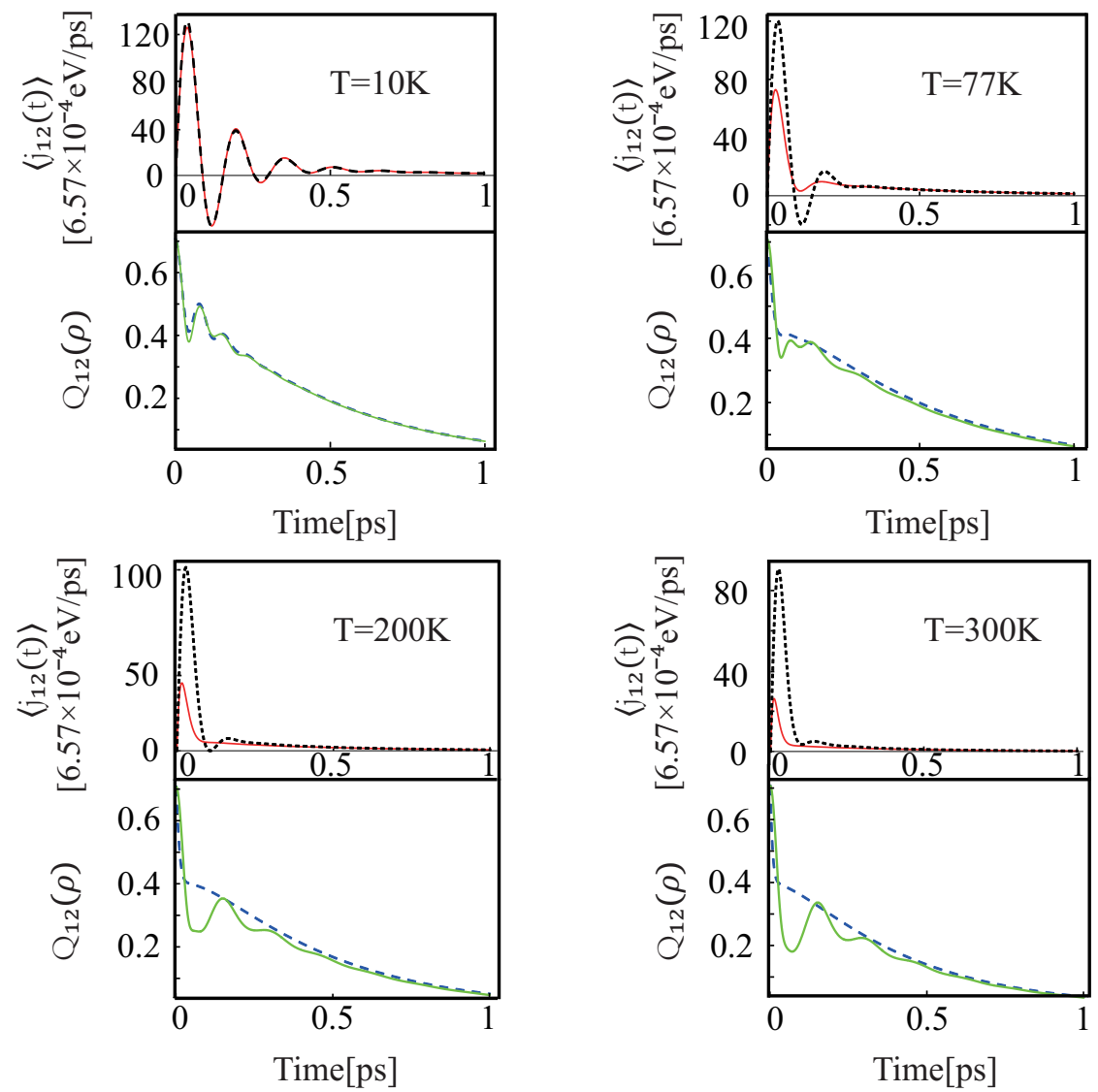

FIG. 7: (Color online) Energy current $\left\langle j_{12}(t)\right\rangle$ (top) and corresponding quantumness $Q_{12}(\rho)$ (bottom) for the case of Markovian (red-solid for $\left\langle j_{12}(t)\right\rangle$ and blue-dashed for $\left.Q_{12}(\rho)\right)$ and non-Markovian (black-dashed for $\left\langle j_{12}(t)\right\rangle$ and green-solid for $Q_{12}(\rho)$ ) dynamics with different values of temperature $T$. Dissipation rate $\Gamma_{1,2}$, trapping rate $\Gamma_{s}$, spectral density parameters $\lambda$, $\omega_{c}$, in the excitonic Hamiltonian take the same values as in Fig. 4

where $\|A\|$ is the Hilbert-Schmidt distance $\|A\|=$ $\left(\operatorname{tr} A A^{\dagger}\right)^{1 / 2}$. Note that this measure of quantumness is introduced to characterize the bipartite quantum correlations between spatially separated sites $|1\rangle$ and $|2\rangle$, not the correlation between the system and environment $[24,[44,50]$. The effect of the environment on the quantumness is included in the density matrix $\rho$ given by Eq. (17). According to this definition, $Q_{12}(\rho) \geq 0$ where $Q_{12}(\rho)=0$ if $\rho$ is classical. An upper bound is given by $Q_{12}(\rho) \leq Q_{\max } \equiv \sqrt{\operatorname{tr} \rho^{2}-1 / d}$, with $d$ the dimension of Hilbert space [44]. In our system, $Q_{\max } \simeq 0.707$. A finite value of $Q_{12}(\rho)$ indicates that there are coherences left in $\rho(t)$ written in the pointer basis. We numerically calculated the quantumness $Q_{12}(\rho)$ for the current $\left\langle j_{12}(t)\right\rangle$ at different temperatures for both Markovian and nonMarkovian dynamics, and present the results in Fig. 7 , where $Q_{12}(\rho)$ is in unit of 1. Either, we don't consider the influence of $\varphi$, as we choose $\rho_{11}(0)=1$ as the initial state.

Similarly, the quantumness $Q_{12}(\rho)$ shows oscillations at the beginning of evolution, especially for lower temperature and non-Markovian case, but it decays fast at higher temperature. This also can be understood as rapid disappearances of the coherent oscillations. It is notable that $Q_{12}(\rho)$ decreases much slower than the oscillation of the energy current. From Fig. 7, for $T=10$ $\mathrm{K}, Q_{12}(\rho)$ drops from its initial value $\left(Q_{\max } \simeq 0.707\right)$ to $Q_{12} \simeq 0.3-0.4$ within $\sim 200 \mathrm{fs}$, but slowly drops to $Q_{12} \simeq 0.1$ at $\sim 800$ fs. From Fig. 4 and Fig. 5, the population and the off-diagonal elements of the density matrix does not exhibit coherent features at $\sim 800 \mathrm{fs}$, while the superposition of the pointer states of the energy current operator retains substantially coherent, comparing with the quantumness of the thermal equilibrium state $\rho_{T}=\exp \left(-H_{S} / k_{B} T\right)$ at the four values of temperature adopted above, which all go to zero as the irreversible loss of energy to the sink and environment. That is to say, energy or exciton transfers through the network to a large extend in the form of a coherent superposition of pointer states of the energy current operator. Quantum coherence plays a significant role in achieving the highly efficient EET. 11 13]. 


\section{CONCLUSION}

In conclusion, we have studied the quantum dynamics of EET through a complex quantum network. We show how the coherent evolution and the environment-induced decoherence, especially the relaxation, work together for the efficient EET. The effect of the phase factor in the coupling constant on the EET depends on initial states, e.g., when the exciton is initially in site $|1\rangle$, the phase factor $\varphi$ has no effect on the transfer efficiency, but when the initial state is a superposition of $|1\rangle$ and $|2\rangle, \varphi$ really has effect on the efficiency. Using the Lindblad master equation, we evaluate the time evolution of population on each site for both Markovian and non-Markovian cases, we find that the dynamics depends on the Markovianity of the system but the efficiency is robust against the parameter variations of the environment. Finally, we quantified the quantum nature of the EET dynamics by calculating the energy current in the network and its quantumness, which helps understand the EET process further. It has been found that the energy current manifests substantial quantumness in both Markovian and non-Markovian dynamics. So, to some extend, we can say the EET dynamics is coherent despite its coupling to environments.

\section{ACKNOWLEDGMENTS}

This work is supported by the NSF of China under Grants No 11175032.

\section{Appendix A: The derivation of Eq. (11)}

In terms of the density matrix elements in the site basis $\rho_{i j}(t)$, the equation of motion are

$$
\begin{aligned}
\dot{\rho}_{11}(t)= & -i\left(J e^{-i \varphi} \rho_{21}-J e^{i \varphi} \rho_{12}\right) \\
& -\Gamma_{1} \rho_{11}-\Gamma_{12} \rho_{11}+\Gamma_{21} \rho_{22}, \\
\dot{\rho}_{22}(t)= & -i\left(J e^{i \varphi} \rho_{12}-J e^{-i \varphi} \rho_{21}\right) \\
& -\Gamma_{2} \rho_{22}+\Gamma_{12} \rho_{11}-\Gamma_{21} \rho_{22}-\Gamma_{s} \rho_{22}, \\
\dot{\rho}_{33}(t)= & \Gamma_{s} \rho_{22}, \\
\dot{\rho}_{00}(t)= & \Gamma_{1} \rho_{11}+\Gamma_{2} \rho_{22}, \\
\dot{\rho}_{12}(t)= & -i\left[-2 \Delta \rho_{12}+J e^{-i \varphi}\left(\rho_{22}-\rho_{11}\right)\right] \\
& -\frac{1}{2}\left(\Gamma_{1}+\Gamma_{2}+\gamma_{1}+\gamma_{2}+\Gamma_{12}+\Gamma_{21}+\Gamma_{s}\right) \rho_{12},
\end{aligned}
$$

where $\Delta=\left(\varepsilon_{2}-\varepsilon_{1}\right) / 2$, and the initial conditions are

$$
\rho_{11}(0)=1, \rho_{00}(0)=\rho_{22}(0)=\rho_{33}(0)=\rho_{12}(0)=0 .(\mathrm{A} 2)
$$

By means of Laplace transform, the coupled differential equations can be converted into a set of algebraic equations for the Laplace s-domain variables,

$$
\begin{aligned}
s \tilde{\rho}_{11}= & -i\left(J e^{-i \varphi} \tilde{\rho}_{21}-J e^{i \varphi} \tilde{\rho}_{12}\right) \\
& -\Gamma_{1} \tilde{\rho}_{11}-\Gamma_{12} \tilde{\rho}_{11}+\Gamma_{21} \tilde{\rho}_{22}+1 \\
s \tilde{\rho}_{22}= & -i\left(J e^{i \varphi} \tilde{\rho}_{12}-J e^{-i \varphi} \tilde{\rho}_{21}\right) \\
& -\Gamma_{2} \tilde{\rho}_{22}+\Gamma_{12} \tilde{\rho}_{11}-\Gamma_{21} \tilde{\rho}_{22}-\Gamma_{s} \tilde{\rho}_{22}, \\
s \tilde{\rho}_{33}= & \Gamma_{s} \tilde{\rho}_{22} \\
s \tilde{\rho}_{00}= & \Gamma_{1} \tilde{\rho}_{11}+\Gamma_{2} \tilde{\rho}_{22}, \\
s \tilde{\rho}_{12}= & -i\left[-2 \Delta \tilde{\rho}_{12}+J e^{-i \varphi}\left(\tilde{\rho}_{22}-\tilde{\rho}_{11}\right)\right] \\
& -\frac{1}{2}\left(\Gamma_{1}+\Gamma_{2}+\gamma_{1}+\gamma_{2}+\Gamma_{12}+\Gamma_{21}+\Gamma_{s}\right) \tilde{\rho}_{12} .
\end{aligned}
$$

From Eq. A3 , we can easily obtain the expression of $\tilde{\rho}_{33}(s)$, and the relation of the Laplace transform for $s$ and $t$ gives

$$
P=\rho_{33}(\infty)=\lim _{s \rightarrow 0} s \tilde{\rho}_{33}(s) .
$$

Then we obtain the expression of Eq. (11). Eq. (10), Eq. (13), and Eq. (16), Eq. (14) can be got in the same way.

\section{Appendix B: Derivative of Eq. (10) with respect to $\Gamma_{s}$}

Now we take the derivative of Eq. (10), and obtain

$$
\frac{d P}{d \Gamma_{s}}=\frac{B+C \Gamma_{s}+D \Gamma_{s}^{2}}{E}
$$

where

$$
\begin{aligned}
C= & 2 \Gamma \Gamma_{12}\left(8 J^{2}+\left(\Gamma+\Gamma_{12}+\Gamma_{21}\right),\right. \\
D= & \Gamma\left(-4 J^{2}+\Gamma_{12}\left(\Gamma+\Gamma_{12}+\Gamma_{21}\right)\right), \\
E= & {\left[\left(4 J^{2}+A \Gamma_{12}\right)\left(2 \Gamma+\Gamma_{s}\right)\right.} \\
& \left.+A \Gamma\left(\Gamma+\Gamma_{s}-\Gamma_{12}+\Gamma_{21}\right)\right]^{2}, \\
A= & 2 \gamma+2 \Gamma+\Gamma_{s}+\Gamma_{12}+\Gamma_{21},
\end{aligned}
$$

and $B>0$ is a very complex expression without $\Gamma_{s}$. From these expressions, the denominator $E>0$. In the numerator, $C>0$ and from the quadratic coefficient $D=$ $\Gamma\left(-4 J^{2}+\Gamma_{12}\left(\Gamma+\Gamma_{12}+\Gamma_{21}\right)\right)$, when $J^{2}<\Gamma_{12}\left(\Gamma+\Gamma_{12}+\right.$ $\left.\Gamma_{21}\right), D>0$, thus $P$ is a monotonic function of $\Gamma_{s}$. when $J^{2}>\Gamma_{12}\left(\Gamma+\Gamma_{12}+\Gamma_{21}\right), D<0$, then $P$ is not a monotonic function of $\Gamma_{s}$, and it increases first and then decreases. 
Opin. Struct. Biol. 16, 654 (2006)

[3] J. Gilmore and R. H. McKenzie, J. Phys. Chem. A 112 , 2162 (2008).

[4] G. D. Scholes, G. R. Fleming, A. Olaya-Castro, and P. V. Grondelie, Nat. Chem. 3, 763 (2011); Y. C. Cheng and G. R. Fleming, Annu. Rev. Phys. Chem. 60, 241 (2009).

[5] R. V. Grondelle and V. I. Novoderezhkin, Nature (London) 463, 614 (2010); R. V. Grondelle, J. P. Dekker, T. Gillbro, and V. Sundstrom, Biochim. Biophys. Acta 1187, 1 (1994).

[6] M. K. Sener, J. D. Olsen, C. N. Hunter, and K. Schulten, Proc. Natl. Acad. Sci. USA 104, 15723 (2007).

[7] G. S. Engel, T. R. Calhoun, E. L. Read, T. K. Ahn, T. Manal, Y. C. Cheng, R. E. Blankenship, and G. R. Fleming, Nature (London) 446, 782 (2007).

[8] H. Lee, Y. C. Cheng, and G. R. Fleming, Science 316, 1462 (2007).

[9] E. Collini, C. Y. Wong, K. E. Wilk, P. M. G. Curmi, P. Brumer, and G. D. Scholes, Nature (London) 463, 644 (2010).

[10] G. Panitchayangkoon, D. Hayes, K. A. Fransted, J. R. Caram, E. Harel, J. Wen, R. E. Blankenship, and G. S. Engel, Proc. Natl. Acad. Sci. USA 107, 12766 (2010).

[11] H. van Amerongen, L. Valkunas, and R. van Grondelle, Photosynthetic Excitons (World Scientific, Singapore, 2000).

[12] F. Caruso, A. W. Chin, A. Datta, S. F. Huelga, and M. B. Plenio, J. Chem. Phys. 131, 105106 (2009).

[13] A. Ishizaki and G. R. Fleming, Proc. Natl Acad. Sci. USA 106, 17255 (2009).

[14] M. B. Plenio and S. F. Huelga, New J. Phys. 10, 113019 (2008).

[15] Bao-quan Ai and Shi-Liang Zhu, Phys. Rev. E 86, 061917 (2012)

[16] S. Hoyer, M. Sarovar, and K. B. Whaley, New J. Phys. 12, 065041 (2010); F. Fassioli and A. Olaya-Castro, New J. Phys. 12, 085006 (2010).

[17] F. Caruso, S. Montangero, T. Calarco, S. F. Huelga, and M. B. Plenio, Phys. Rev. A 85, 042331 (2012).

[18] A. W. Chin, A. Datta, F. Caruso, S. F. Huelga, and M. B. Plenio, New J. Phys. 12, 065002 (2010).

[19] Y. C. Cheng and R. J. Silbey, Phys. Rev. Lett. 96, 028103 (2006).

[20] A. Nazir, Phys. Rev. Lett. 103, 146404 (2009).

[21] T. R. Calhoun, N. S. Ginsberg, G. S. Schlau-Cohen, Y. C. Cheng, M. Ballottari, R. Bassi, and G. R. Fleming, J. Phys. Chem. B 113, 16291 (2009).

[22] P. Rebentrost, M. Mohseni, I. Kassal, S. Lloyd, and A. Aspuru-Guzik, New J. Phys. 11, 033003 (2009); P. Rebentrost, M. Mohseni, and A. Aspuru-Guzik, J. Phys. Chem. B 113, 9942 (2009).

[23] B. Cui, X. X. Yi, and C. H. Oh, J. Phys. B: At. Mol. Opt. Phys. 45, 085501 (2012); B. Cui, X. Y. Zhang, and X. X. Yi, e-print arXiv:1106.4429.

[24] M. Sarovar, A. Ishizaki, G. R. Fleming, and K. Birgitta Whaley, Nature Physics 6, 462 (2010).

[25] A. Ishizaki and G. R. Fleming, J. Chem. Phys. 130, 234111 (2009).

[26] S. Yang, D. Z. Xu, Z. Song, and C. P. Sun, J. Chem. Phys. 132, 234501 (2011).
[27] P. K. Ghosh, A. Y. Smirnov and F. Nori, J. Chem. Phys. 134, 244103 (2011).

[28] J. Prior, A. W. Chin, S. F. Huelga, and M. B. Plenio, Phys. Rev. Lett. 105, 050404 (2010).

[29] J. Adolphs and T. Renger, Biophys. J. 91, 2778 (2006).

[30] J. S. Cao and R. J. Silbey, J. Phys. Chem. A 113, 13825 (2009); A. Kelly and Y. M. Rhee, J. Phys. Chem. Lett. 2, 808 (2011).

[31] A. A. Faisal, L. P. J. Selen and D. M. Wolpert, Nat. Rev. Neurosci. 9, 292 (2008).

[32] M. Mohseni, P. Rebentrost, S. Lloyd, and A. AspuruGuzik, J. Chem. Phys. 129, 174106 (2008).

[33] X. X. Yi, X. Y. Zhang, and C. H. Oh, Eur. Phys. J. D 67, $172(2013)$.

[34] H. Haken and G. Strobl , Z. Phys. 262135 (1973).

[35] P. Rebentrost, R. Chakraborty, and A. Aspuru-Guzik, J. Chem. Phys. 131, 184102 (2009).

[36] F. Fassioli and A. Olaya-Castro, New J. Phys. 12, 085006 (2010).

[37] Q.-S. Tan and L.-M. Kuang, Commun. Theor. Phys. 58, 359 (2012).

[38] J.-Q. Liao, J.-F. Huang, L.-M. Kuang, and C. P. Sun, Phys. Rev. A 82, 052109 (2010).

[39] H. J. Briegel and S. Popescu, e-print arXiv:0806.4552.

[40] J. Cai, G. G. Guerreschi, and H. J. Briegel, Phys. Rev. Lett. 104, 220502 (2010).

[41] M. Thorwart, J. Eckel, J. H. Reina, P. Nalbach, and S. Weiss, Chem. Phys. Lett. 478, 234 (2009).

[42] F. Caruso, A. W. Chin, A. Datta, S. F. Huelga, and M. B. Plenio, Phys. Rev. A 81, 062346 (2010).

[43] A. Olaya-Castro , C. F. Lee , F. F. Olsen, and N. F. Johnson, Phys. Rev. B 78, 085115 (2008).

[44] O. Giraud, P. Braun, and D. Braun, New J. Phys. 12, 063005 (2010).

[45] W. H. Zurek, Phys. Rev. D 24, 1516 (1981).

[46] W. H. Zurek, Rev. Mod. Phys. 75, 715 (2003).

[47] J. Yuen-Zhou, S. K. Saikin, N. Yao, and A. AspuruGuzik, e-print arXiv:1406.1472.

[48] F. Fassioli, A. Nazir, and A. Olaya-Castro, J. Phys. Chem. Lett. 1, 2139 (2010).

[49] H.-P. Breuer and F. Petruccione, The Theory of Open Quantum Systems (Oxford University Press, Oxford, 2007).

[50] P. Nalbach, D. Braun, and M. Thorwart, Phys. Rev. E 84, 041926 (2011).

[51] O. Giraud, P. Braun, and D. Braun, Phys. Rev. A 78, 042112 (2008).

[52] J. Martin, O. Giraud, P. A. Braun, D. Braun, and T. Bastin, Phys. Rev. A 81, 062347 (2010).

[53] R. J. Hardy, Phys. Rev. 132, 168 (1963).

[54] J. Deppe and J. L. Feldman, Phys. Rev. B 50, 6479 (1994).

[55] D. Segal, A. Nitzan, and P. Hanggi, J. Chem. Phys. 119, 6840 (2003).

[56] P. B. Allen and J. L. Feldman, Phys. Rev. B 48, 12581 (1993).

[57] D. M. Leitner, J. Chem. Phys. 130, 195101 (2009).

[58] L. Wu and D. Segal, J. Phys. A 42 , 025302 (2009). 